\title{
Mode identification of multi-periodic Slowly Pulsating B-stars: results and problems
}

\author{
W. Zima, ${ }^{1}$ P. De Cat ${ }^{2}$ C. Aerts ${ }^{1}$ \\ ${ }^{1}$ Instituut voor Sterrenkunde, K.U. Leuven, Celestijnenlaan 200D, 3001 Leuven, Belgium \\ ${ }^{2}$ Koninklijke Sterrenwacht van België, Ringlaan 3, 1180 Brussel, Belgium
}

\begin{abstract}
We report results from mode identifications (MI) for four selected multi-periodic Slowly Pulsating B-stars (SPB) using the Fourier parameter fit (FPF) method. Problems related to the pulsational nature of these objects which hamper a correct identification are discussed. For a present day status of knowledge about SPB stars we refer to De Cat (2007, this issue).
\end{abstract}

\section{Introduction}

De Cat et al. (2000), De Cat (2001), and De Cat \& Aerts (2002, hereafter DA02) carried out a large photometric and spectroscopic monitoring program including 13 SPB stars to provide a better understanding for this class of variables. Their frequency analysis and mode identification are based on 7-colour Geneva-photometry, Hipparcos measurements, and timeseries of high-resolution, high signal-to-noise spectra. We selected four multi-periodic SPB stars, HD 26326, HD 74195, HD 85953, and HD 138764, which are promising targets for successful seismic modelling, to perform MI. We applied the Fourier parameter fit (FPF) method (Zima 2006) to their data by which we fit the observed zeropoint, amplitude and phase across an absorption line profile for each detected frequency with corresponding values from synthetic line profiles to determine the pulsational geometry. For each separate case we selected the deepest unblended line of the SiII-triplet.

\section{Results}

HD 26326: DA02 detected three frequencies from multi-colour photometry and the first three normalized velocity moments of the $\lambda 4128 \AA$ Sili profiles: $0.534,0.172$ and $0.763 \mathrm{~d}^{-1}$. By using amplitude ratios (Dupret et al. 2003) in seven passbands of the Geneva photometric system, the dominant frequency $f_{1}$ was identified as $\ell=1$ or 2 (De Cat et al., in preparation). By applying a Fourier analysis and least-squares fitting for each pixel across the $\lambda 4128 \AA$ SiII profile we detected $f_{1}$ and $f_{2}$ in agreement with DA02, but the value of a third frequency $\left(f_{3}=0.7559 \mathrm{~d}^{-1}\right)$ differs significantly for reasons unknown, yet. We identified $f_{1}$ with high significance as a prograde sectoral dipole-mode $(\ell=m=1)$ and constrained the azimuthal order for $f_{3}$ to $m=1$. For $f_{2}$, the results of the $\mathrm{Ml}$ are ambiguous.

HD 74195: For this object, DA02 report four frequencies between 0.3 and $0.4 \mathrm{~d}^{-1}$. All four periodicities can be seen in the velocity moments with radial velocity amplitudes between 1.3 and $3.2 \mathrm{~km} \mathrm{~s}^{-1}$. We detected only two of them in the intensity variations across the $\lambda 4128 \AA$ SiII profile: $f_{1}=0.357 \mathrm{~d}^{-1}$ and $f_{2}=0.350 \mathrm{~d}^{-1}$. We identified the azimuthal order of $f_{1}$ as $m=1$, but an ambiguity in the determination of $\ell$ remained. By also considering the photometric MI $(\ell=1)$ we conclude that this is a sectoral dipole-mode. The best fits for $f_{2}$ are achieved with $(\ell, m)=(3,-2)$ and $(3,+1)$, not in agreement with the photometric MI of $\ell=1$. 
HD 85953: The mean line profile shows a clear asymmetry which may be caused by a composite profile due to a visual component. We therefore carried out the $\mathrm{MI}$ in two steps: first by considering the zeropoint profile, which sets strong constraints on $v \sin i$, the intrinsic line width and the equivalent width, and second by only fitting amplitude and phase across the profile and omitting the zeropoint profile. It turned out that for both frequencies a better fit can be achieved with the second approach resulting in a narrower zeropoint profile and a lower $v \sin i$ value of $18 \mathrm{~km} \mathrm{~s}^{-1}$, compared to $29 \mathrm{~km} \mathrm{~s}^{-1}$ when considering the zeropoint profile. This might indicate that we are indeed dealing with a composite profile which increases the width of the line. The best identification for $f_{2}$ yields $(\ell=4, m=-3)$. For $f_{1}$ large ambiguities prevent a clear identification.

HD 138764: This star is multi-periodic with at least two periodicities (DA02). A dominant frequency at $0.794 \mathrm{~d}^{-1}$ with a radial velocity amplitude of $3.6 \mathrm{~km} \mathrm{~s}^{-1}$ is found from photometry and spectroscopy. The velocity moments show a second frequency at $0.637 \mathrm{~d}^{-1}$ with a much lower radial velocity amplitude of $0.8 \mathrm{~km} \mathrm{~s}^{-1}$. Our analysis of the pixel-intensity variations of the $\lambda 4130 \AA$ SiII line revealed $f_{1}=0.794 \mathrm{~d}^{-1}, f_{2}=0.637 \mathrm{~d}^{-1}$ and an additional frequency at $f_{3}=0.589 \mathrm{~d}^{-1}$. By means of the FPF method $f_{1}$ is clearly identified as a sectoral mode with $\ell=1, m=1$, which is in good agreement with the photometric results and the identification from the moment method (De Cat et al., in preparation). The best fit to the Fourier parameters of this mode indicates an inclination angle of $30^{\circ}$ and a $v \sin i$ of $21 \mathrm{~km} \mathrm{~s}^{-1}$, implying an equatorial rotational velocity of $42 \mathrm{~km} \mathrm{~s}^{-1}$ and a rotational frequency of $0.32 \mathrm{~d}^{-1}$. The amplitude and phase of $f_{2}$ and $f_{3}$ across the profile are strongly distorted due to the relatively large amplitude of $f_{1}$, therefore no reliable MI could be achieved for these two modes.

\section{Conclusions}

Mode identification of SPB stars is very challenging due to the large horizontal velocities, which hamper the identification of the azimuthal order of the pulsation modes. For some pulsation modes $\ell$ and $m$ can be constrained but the uncertainty in the determination of the stellar inclination is for most stars too large to derive reliable frequency values in the stellar rotation frame of reference. The knowledge of these frequency values are very important for theoretical modelling. All identifications point towards low-degree and low-order pulsation modes. In many cases the dominant frequency is a sectoral dipole-mode. Recently, Hubrig et al. (2006) detected longitudinal magnetic fields with strengths up to a few hundred $G$ for a set of SPB stars. An improved model of the line profile variations by also considering the effects of a magnetic field and non-uniform surface element abundances will be necessary for a better understanding of these objects.

Acknowledgments. WZ and CA are supported by the Research Council of Leuven University, under grant GOA/2003/04.

\section{References}

De Cat P., Aerts C., De Ridder J., et al., 2000, A\&A, 355, 1015

De Cat P., 2001, PhD Thesis, Katholieke Universiteit Leuven, Belgium

De Cat P., Aerts C., 2002, A\&A, 393, 965

Dupret M.-A., De Ridder J., De Cat P., et al., 2003, A\&A, 398, 677

Hubrig S., Briquet M., Schöller M., et al., 2006, MNRAS, 369, 61

Zima W., 2006, A\&A, 455, 227 